\title{
Table ronde 2
}

\section{Les évolutions suite \\ aux décisions de la loi \\ de sécurité sanitaire - \\ Conséquences en France}

Participent à la table ronde:

Anne d'Andon, Haute autorité de santé (HAS)

Marie-Christine Borrelly, Octapharma

Nadine David, Direction générale de la santé, Bureau des médicaments

Christophe Duguet, AFM-Téléthon

Annie Lorence, Agence nationale de sécurité du médicament (ANSM)

Faisant suite à la crise du Mediator ${ }^{\circledR}$, cette loi était articulée autour de trois axes principaux: assurer la transparence des liens d'intérêt; organiser une refonte de la gouvernance des produits de santé avec la création de l'Agence nationale de sécurité du médicament et des produits de santé (ANSM) ; améliorer l'évaluation des produits de santé après leur mise sur le marché et proposer un nouvel encadrement pour les situations de prescription hors AMM ou sans AMM. Cette vaste loi a fait l'objet de 21 décrets d'application.

Pour cette table ronde, nous avons choisi de nous attacher tout particulièrement aux situations de prescription hors AMM ou sans AMM, et aux dispositions législatives afférentes.

\section{Nadine David}

L'affaire du Mediator ${ }^{\circledR}$ a mis en évidence un certain nombre de dysfonctionnements en termes de sécurité sanitaire. Ce médicament est indiqué dans le traitement du diabète. La Caisse nationale d'assurance maladie (CNAM) a identifié que certains patients traités par Mediator ${ }^{\circledR}$ ne recevaient pas d'autre traitement antidiabétique. $30 \%$ des prescriptions de ce médicament étaient hors AMM (ce taux est monté à $70 \%$ dans les dernières années de prescription).

Par ailleurs, une pneumologue de Brest a réalisé une étude mettant en évidence le lien entre le Mediator ${ }^{\circledR}$ et l'hypertension artérielle pulmonaire dont souffraient ses jeunes patientes. D'autres études ont objectivé le lien entre la prise de Mediator ${ }^{\circledR}$ et la survenue de pathologies cardio-vasculaires. Le médicament s'est vu retirer son AMM en novembre 2009.

Suite à cela, plus de 8000 plaintes de patients ayant reçu le Mediator ${ }^{\circledR}$ et qui présentaient des pathologies cardio-vasculaires ont été déposées, suscitant alors de larges débats, qui ont par la suite été repris dans les médias. Cette affaire a créé un climat de suspicion

généralisée. Que savait-on sur la dangerosité du Mediator ${ }^{\circledR}$ ? Pourquoi I'AMM n'a-t-elle pas été retirée plus tôt ? Comment les médecins ontils été informés de l'effet anorexigène du Mediator ${ }^{\circledR}$ ? Pourquoi l'ontils prescrit hors AMM ? Savaient-ils qu'ils le prescrivaient hors AMM? Plus généralement, cette affaire a généré une suspicion sur la sécurité des autres médicaments. De nombreux travaux d'évaluation et de concertation ont été menés pour proposer une amélioration du système de sécurité sanitaire du médicament.

Deux rapports de l'Inspection générale des affaires sociales (IGAS), un rapport de l'Assemblée nationale, un rapport du Sénat et les Assises du médicament ont recommandé des mesures pour mieux protéger les patients, notamment en termes d'encadrement hors AMM, d'un meilleur suivi des patients en pré-AMM et en hors AMM, de meilleures formations et informations des médecins, et d'une meilleure information des patients (avec la mise en place récente de la base de données des médicaments).

La prescription hors AMM est de l'ordre de $20 \%$. Ce taux peut atteindre $80 \%$ en pédiatrie, en cancérologie et dans les maladies rares. La prescription hors AMM est justifiée lorsqu'il y a un décalage entre les connaissances du moment et les données de l'AMM. En revanche le hors AMM injustifié doit être encadré, car il peut être dangereux. Des mesures ont été portées par le Gouvernement dans le cadre 
de la loi Bertrand. Les objectifs de cette loi sont de sécuriser les patients, en évitant les prescriptions dangereuses et en améliorant les connaissances pour aboutir à une extension d'AMM.

$\varepsilon$ France, les médecins bénéficient de la liberté de prescription. Pour la première fois, la loi Bertrand est venue encadrer cette liberté. La loi stipule en effet qu'une prescription hors AMM ne peut se faire que lorsqu'il n'existe pas d'alternative et lorsqu'il existe une recommandation spécifique de l'ANSM ou que le prescripteur juge que le traitement est indispensable pour le patient au regard de la science. Le prescripteur doit alors informer le patient que la prescription est hors AMM, et qu'il n'existe pas d'alternative; en outre, il doit écrire «hors $A M M$ » sur l'ordonnance.

De leur côté, les entreprises du médicament doivent s'engager à contribuer au bon usage du médicament. Lorsqu'elles constatent des prescriptions non conformes au bon usage, elles doivent d'une part informer les professionnels en cas de prescription hors AMM dangereuse, et d'autre part informer l'ANSM qui par la suite évaluera le hors AMM.

\section{Chrystel Jouan-Flahault}

Mme Lorence va nous présenter les différents types de situation n'entrant pas dans le cadre d'une AMM.

\section{Annie Lorence}

Je vais vous présenter le dispositif des ATU et des RTU, qui sont deux missions dévolues à I'ANSM. En matière de mise à disposition des médicaments, I'AMM correspond au «gold standard». Lorsque le médicament ne dispose pas d'une AMM, il peut être mis à disposition des patients dans le cadre d'une ATU. Lorsque le médicament dispose d'une AMM, il peut être prescrit hors AMM, soit dans le cadre d'une RTU, soit sous la seule responsabilité du prescripteur sur la base de données dont il dispose. Dans le même contexte d'absence d'alternative médicamenteuse, des préparations peuvent être prescrites aux patients.

Le dispositif des ATU existe depuis 1994. La loi Bertrand a introduit des nouveaux critères, notamment en matière de recevabilité des ATU. II existe deux types d'ATU : les ATU de cohorte (ATUC) et les ATU nominatives (ATUn). Ce dispositif exceptionnel de mise à disposition de médicament sans AMM est destiné au traitement de maladies graves ou rares, pour lesquelles il existe un besoin thérapeutique - c'est-àdire qu'il n'existe pas de traitement autorisé approprié et disponible en France. En outre, la loi Bertrand introduit le fait que le traitement ne peut pas être différé.

Les ATUn sont octroyées à la demande et sous la responsabilité d'un médecin hospitalier pour un patient nommément désigné qui ne peut pas être inclus dans une recherche clinique, et pour lequel il existe des données d'efficacité/sécurité qui permettent de présumer d'un rapport bénéfice/risque favorable. La nouveauté introduite par la loi Bertrand est qu'une demande d'ATUn n'est recevable que dans deux cas : soit le médicament fait l'objet d'une demande d'AMM ou d'ATUc par un laboratoire, ou à défaut celui-ci s'engage à faire cette demande dans un délai déterminé ; soit le médicament fait l'objet d'un essai clinique en France ou va faire l'objet d'une demande d'autorisation d'essai clinique.
L'ANSM peut toutefois délivrer des ATUn dans les cas suivants :

- si des conséquences graves à court terme pour le patient sont très fortement probables ;

- si le médicament fait l'objet d'un arrêt de commercialisation, mais qu'il existe un besoin pour certains patients dans une autre indication (comme le Nizoral ${ }^{\circledR}$ dans la maladie de Cushing);

- si le médicament s'est fait refuser une ATUc ou un essai clinique, mais qu'il existe un bénéfice individuel pour le patient.

L'ATUc est octroyée à la demande d'un laboratoire pour un groupe de patients sur la base de données d'efficacité/sécurité permettant de présumer fortement d'un rapport bénéfice/risque favorable. En outre, le laboratoire s'engage à faire le nécessaire pour obtenir I'AMM. L'ATUc est octroyée pour un an, renouvelable si besoin; elle s'accompagne d'un résumé des caractéristiques du produit (RCP), d'une notice et d'un étiquetage en français, ainsi que d'un protocole d'utilisation thérapeutique (PUT) obligatoire.

L'ATUn est octroyée pour la durée du traitement envisagée pour le patient; et peut être renouvelée le cas échéant. Le PUT est désormais obligatoire pour les ATUn, sauf pour les trois situations « dérogatoires » précitées. Ce PUT permet le recueil de données d'efficacité/sécurité, données importantes dans le domaine des maladies rares - il s'agit là d'une des demandes du PNMR.

Dans les 2 types d'ATU, le patient doit être informé des modalités de mise à disposition du médicament - le médecin les inscrira dans le dossier médical patient.

L'objectif de la loi Bertrand est également de promouvoir les ATUc en remplacement des ATUn.

$25 \%$ des ATU actuelles concernent des médicaments orphelins.

Concernant les médicaments disposant d'une AMM, ils peuvent être prescrits en dehors de leur AMM si une RTU est établie par l'ANSM (nouveau dispositif) ou à défaut si le prescripteur juge cette prescription indispensable au regard des données acquises de la science.

Pour rappel, des textes relatifs au hors AMM existaient déjà, mais leur objectif était de permettre le remboursement. Il s'agit du décret $n^{\circ} 2005-1023$ du 24 août 2005 (modifié en 2008) qui est relatif au contrat de bon usage des médicaments et des produits et prestations mentionnés à l'article L. 162-22-7 du code de la sécurité sociale (décret pour les médicaments onéreux). Le décret $n^{\circ} 2012-740$ du 9 mai 2012 permet la prise en charge dérogatoire par l'assurance maladie de spécialités pharmaceutiques prescrites dans le domaine des maladies rares ou affections de longue durée et bénéficiant d'une RTU. 
Ce nouvel encadrement du hors AMM vise à renforcer la sécurité d'utilisation des médicaments dans l'intérêt des patients, via une information et un suivi des patients. De plus, l'élaboration de RTU permet une égalité d'accès aux traitements.

Lorsque I'ANSM élabore une RTU, celle-ci doit inclure plusieurs mentions (indication dérogatoire, posologie et mode d'administration; si besoin, contre-indications, mises en garde, précautions d'emploi, effets indésirables; le cas échéant, les conditions de prescription de délivrance différentes de celles de I'AMM d'origine). La RTU s'accompagne d'un argumentaire sur les données disponibles permettant de présumer d'un rapport bénéfice/risque favorable.

En outre, la RTU contient en annexe un protocole de suivi. Elle permet, via des fiches de suivi, de recueillir des données d'efficacité/sécurité et de conditions réelles d'utilisation du médicament dans l'indication visée par la RTU. Ces fiches de suivi - qui ne remplaceront pas l'essai clinique - seront collectées par les laboratoires et utilisées en vue de la rédaction de rapports de synthèse périodiques qui permettront à l'ANSM de vérifier que le rapport bénéfice/risque est toujours favorable. Ce suivi est obligatoire et doit être financé par le(s) laboratoire(s) concerné(s).

Enfin, la RTU peut s'accompagner d'une convention entre l'ANSM et les laboratoires concernés, avec un possible engagement de ces derniers à demander une modification d'AMM dans un délai fixé.

L'ANSM dispose aujourd'hui d'un certain nombre de signalements issus essentiellement de l'enquête DGS/DGOS réalisée en 2012 auprès des centres de référence maladies rares. 69 centres de référence ont répondu et transmis 550 signalements dans de nombreux domaines thérapeutiques et de qualité très hétérogène. L'ANSM effectue actuellement un travail de tri pour permettre d'identifier les sujets pouvant faire l'objet de RTU. Par ailleurs, la réévaluation des protocoles temporaires de traitement (PTT) concernant les maladies rares est en cours.

À ce jour, 9 RTU sont en cours d'évaluation dans les maladies rares. Les RTU n'ont pas vocation à être octroyées dans toutes les situations hors AMM ; elles sont élaborées pour encadrer l'usage de médicaments, notamment lorsqu'un enjeu de santé publique se présente, et nécessitant un suivi particulier; en outre, l'Agence doit disposer de données suffisantes pour présumer du rapport bénéfice/risque favorable.

Les RTU ne remplacent pas les essais cliniques qui sont à promouvoir, notamment dans les nombreuses situations hors AMM pour lesquelles l'Agence ne pourra pas accorder de RTU pour insuffisance de données. Toutes les RTU seront publiées sur le site de l'Agence (www.ansm.sante.fr).

\section{Chrystel Jouan-Flahault}

Anne d'Andon va maintenant nous présenter les dispositifs mis en place suite à la loi sur les médicaments pour les problèmes de prise en charge des patients.

\section{Anne d'Andon}

L'encadrement des prescriptions hors AMM conduit à une situation de prise en charge qui concerne les médicaments qui font l'objet de RTU, puisqu'à l'issue de la recommandation faite par l'ANSM, la HAS doit se prononcer sur le bien-fondé du remboursement de ces médicaments ; elle a aussi la possibilité de se saisir ou d'être saisie sur le bien-fondé de la prise en charge de dispositifs médicaux ou de produits et prestations qui concernent notamment des maladies rares.

Un médecin peut, sur la base des données de la science et des connaissances existantes, considérer qu'il y a matière et assurance d'un intérêt d'un médicament pour un patient. Il peut alors prescrire ce médicament en dehors des indications de l'AMM. Dans ce cas, il se doit d'une part d'informer le malade de cette situation, et d'autre part de lui préciser que le médicament ne sera pas pris en charge.

\section{Chrystel Jouan-Flahault}

Marie-Christine Borrelly va nous faire part du point de vue d'un industriel sur ces nouvelles dispositions législatives.

\section{Marie-Christine Borrelly}

Je m'exprimerai au nom du laboratoire Octapharma, laboratoire de médicaments dérivés du plasma. En France, notre site exploitant emploie 28 personnes, tandis que notre site industriel compte 400 salariés. Notre petit laboratoire de médicaments techniques purement hospitaliers (médicaments dérivés du plasma) a accueilli avec attention la loi Bertrand de 2011. Nous étions en phase avec ses finalités puisque nous ne pouvons être que favorables au bon encadrement des produits de santé. Notre laboratoire a toutefois accueilli cette loi comme un raz-de-marée, à une période où de nouvelles normes étaient adoptées au niveau européen (en pharmacovigilance, par exemple...). En 2012, nous avons vu arriver un certain nombre de décrets, en particulier sur la transparence des liens avec les professionnels de santé, la maîtrise du bon usage, etc. L'implémentation simultanée de ces nouvelles obligations a présenté de réelles difficultés pour notre site exploitant. Nous avons dû augmenter nos effectifs dans le domaine des affaires pharmaceutiques et au niveau administratif. Nous avons investi sur les différentes obligations, en priorisant la mise à jour de la pharmacovigilance, le projet Sunshine $A c t^{1}$, les nouvelles modalités de bonnes pratiques de distribution, et le hors AMM. À l'hôpital, les prescriptions de nos médicaments se font à l'aide de fiches de prescription où les usages AMM et hors AMM peuvent être identifiés. $\varepsilon$ n outre, l'utilisation de ces médicaments fait l'objet d'une traçabilité particulière - par le biais d'étiquettes

\footnotetext{
${ }^{1}$ http://www.lepetitjuriste.fr/droit-civil/droit-medical/sunshine-act-a-la-francaise-volet-transparence-des-liens-entre-professionnels-de-sante-et-industriels-de-produits-de-sante
} 
spécifiques jusqu'au patient. De plus ces médicaments font fréquemment l'objet d'études post-AMM. À l'initiative des centres de référence, des registres sont tenus, notamment dans le cas des inductions de tolérance immunes.

Les prescriptions hors AMM sont encadrées par des protocoles temporaires de traitement (PTT).

De leur côté, les laboratoires déposent des dossiers d'extension d'indications.

Les autres difficultés rencontrées par les laboratoires de notre taille tiennent au nombre de collaborateurs en contact avec les professionnels de santé. Huit personnes de notre site exploitant - qui emploie 28 salariés - sont chargées de l'information médicale. La fréquence de contact avec les cibles est très faible, et la couverture des prescripteurs en France reste problématique.

Par ailleurs, je souhaitais souligner la difficulté d'aborder la notion d'usage hors AMM si le médecin ne l'aborde pas lui-même spontanément, car ce dernier peut alors percevoir cette intervention comme une ingérence, voire une promotion du hors AMM.

Octapharma considère que les quatre sources d'information sur le mésusage sont les suivantes:

- la pharmacovigilance ;

- I'information médicale;

- les réclamations pharmaceutiques;

- la veille bibliographique.

Concernant cette dernière source, la difficulté rencontrée est d'intervenir auprès de l'auteur de l'article ou du poster; expert qui a jugé bon de communiquer à la communauté scientifique un certain point de vue, pour lui rappeler qu'il utilise le produit en dehors de son AMM.

Chez Octapharma, tout le hors AMM tourne autour de la pharmacovigilance, où nous recueillons les informations en provenance des sources précitées. Nous les colligeons, les «trackons » et rappelons aux professionnels de santé les termes de l'AMM en envoyant, lorsque cela est nécessaire, les résumés des caractéristiques du produit (RCP) et les termes de l'AMM validés. Ainsi l'ANSM est informée via la pharmacovigilance. En préventif, Octapharma n'a pas mandaté ses visiteurs médicaux pour systématiquement rechercher des cas de mésusage. En revanche, notre visite médicale a été formée dès le mois d'octobre, via une formation, à la procédure de pharmacovigilance. En outre, nous avons réalisé un formulaire de recueil de prescriptions hors AMM. Pour l'instant, le système ne fonctionne pas encore très bien puisque les retours des visiteurs médicaux restent faibles.

Les constats d'usages hors AMM sont très utiles aux laboratoires, car ils peuvent alors identifier de nouvelles voies de développement et initier des essais de phase III en complément des RTU. Ainsi, nous avons initié un essai de phase III dans la sclérose en plaques en Europe.

Dans d'autres cas, des registres ont été élaborés par des experts en France.

Ainsi, la mise en lumière de l'usage hors AMM présente de nombreuses vertus, tant dans le sens de la sécurité que dans celui du développement, et permet une documentation approfondie de l'usage des spécialités de santé.

\section{Chrystel Jouan-Flahault}

Christophe Duguet va maintenant nous dire si ce dispositif lui paraît adéquat au regard de l'enjeu.

\section{Christophe Duguet}

Lors de RARE 2011, j'avais fait une présentation sur la question du hors $A M M$, en montrant que nous étions dans une zone grise, qui n'est pas apparue avec le Médiator ${ }^{\circledR}$ puisque des dispositifs ont été mis en place ces dernières années pour essayer d'encadrer et reconnaître les PTT et l'article 56 . Ces dispositifs se sont multipliés progressivement pour mieux appréhender le fait que des centaines de milliers de patients utilisent quotidiennement des médicaments hors AMM.

Il y a deux ans, ma présentation se terminait de manière optimiste, car nous venions d'éviter de justesse, après le «tourbillon » Médiator ${ }^{\circledR}$, une interdiction totale du hors AMM. Quelque temps après la promulgation de la loi, Xavier Bertrand, ministre de la Santé, avait expliqué lors d'une conférence de presse qu'il avait décidé d'interdire le hors AMM, mais il était revenu sur sa décision en étudiant les maladies rares. J'étais alors plutôt optimiste puisqu'une discussion avait eu lieu au Parlement sur la spécificité des maladies rares dans la mise en œuvre des RTU. Un amendement du Gouvernement avait en effet pointé les difficultés particulières se posant pour les maladies rares; il proposait donc que soit prise en compte l'expertise des centres de référence maladies rares.

Entre la promulgation de la loi Bertrand en 2011 et aujourd'hui, aucune RTU n'est sortie. Certaines sont en cours d'instruction; une vingtaine d'entre elles concernerait les maladies rares sur plus de 550 signalements remontés par les centres de référence. Aujourd'hui, il n'est pas possible d'envisager d'avoir des RTU pour des utilisations hors AMM, notamment pour des maladies rares, au-delà d'un nombre de situations très limitées, parce que pour de nombreuses utilisations hors AMM très anciennes, nous sommes encore loin du minimum de données nécessaire pour qu'une autorité puisse accepter de donner son aval. C'est pourquoi le système mis en place avec les RTU répond tout à fait correctement à un certain nombre de situations; nous espérons même qu'il sera utile pour accélérer le passage d'utilisations hors AMM vers de l'AMM, car tel est l'objectif de I'ATU. Malheureusement, ce système ne répond pas à une grande majorité de situations qui concernent des centaines de milliers de patients.

Nous pourrions continuer à être dans cette zone grise, empreinte d'une certaine hypocrisie, dans laquelle la loi reconnaît la possibilité pour le prescripteur de prescrire hors AMM au vu des données acquises de 
la science. Si le prescripteur prescrit, l'assurance maladie ne doit normalement pas rembourser le médicament. Dans la majorité des cas, un médicament prescrit qui n'est pas remboursé s'apparente à un médicament non prescrit - sauf si on continue à fermer les yeux. Cette situation n'est pas acceptable, parce que de nombreuses utilisations hors AMM sont tout à fait utiles aux patients, tandis que d'autres utilisations sont probablement inutiles, voire dangereuses. Pour les patients, il est donc impératif de pouvoir sortir de cette situation.

Les parlementaires avaient pointé l'intérêt des centres de référence. L'ensemble des dispositifs a en effet permis de labelliser et d'évaluer la meilleure expertise sur des maladies rares pour le suivi, le soin et la recherche sur les patients. Les centres de référence constituent donc un outil fort. Dans le nouveau système que nous devrons inventer, l'expertise et le consensus d'experts entre les différents centres de référence concernés par les maladies rares devront exercer un poids beaucoup plus important dans l'utilisation des médicaments et le processus dérogatoire permettant leur remboursement, sous réserve que ces utilisations permettent d'acquérir les données nécessaires à une visibilité accrue sur la sécurité, l'efficacité et l'effet des produits. Dans ce système apprenant, l'acquisition progressive de données permettra éventuellement d'aller vers une RTU, voire une AMM.

$\varepsilon$ n outre, ce système devra soutenir les centres de référence en leur apportant un soutien méthodologique - pour une mise en registre de qualité et le suivi de cohorte - et un soutien financier permettant que les utilisations de médicaments hors AMM se fassent de manière sécurisée, tout en assurant l'acquisition de données. La réglementation actuelle ne permet pas de faire cela.

Fin 2014, une loi de santé sera discutée. Elle constituera sans doute une occasion intéressante pour amener les évolutions réglementaires nécessaires et concevoir le système de soutien financier qui pourrait y être associé. Nous pourrions ainsi essayer de sortir de cette zone d'hypocrisie collective, dans laquelle on prescrit, on tolère, on rembourse, et on dénonce les risques sur la sécurité. Au final, on se satisfait d'une situation qui n'est pas satisfaisante.

\section{Chrystel Jouan-Flahault}

Avant de me tourner vers la salle, je souhaiterais passer la parole à Cécile Delval, qui va aborder deux autres conséquences de la loi Bertrand: la gouvernance des produits de santé et la transparence des liens.

\section{Cécile Delval, Directrice de l'évaluation à l'ANSM}

Avant de parler des nouveaux moyens de l'ANSM pour recourir à l'expertise, j'apporterai quelques précisions sur ce qui a été dit sur la place des centres de référence dans le process RTU. Suite à l'enquête faite auprès des centres de référencement, I'ANSM a reçu 550 signalements. $10 \%$ d'entre eux n'étaient pas recevables parce que la demande ne concernait pas un médicament ou parce que le médicament n'avait pas d'AMM.

Pour que I'ANSM instruise une RTU, une présomption de rapport bénéfice/risque favorable est nécessaire.
II convient par ailleurs de noter que l'ANSM est favorable aux évolutions suivantes:

- placer les centres de référence au centre du dispositif ; - encadrer le hors AMM;

- apporter de la méthode pour que les centres de référence remontent des signalements exploitables à I'ANSM.

L'ANSM a adressé un premier courrier de relance aux centres de référence leur demandant la justification du hors AMM, et nous avons reçu des réponses pour $20 \%$ des signalements. Une deuxième relance est en cours. Les résultats de cette enquête seront rendus publics.

Je vais maintenant évoquer la réorganisation de l'Agence suite à la promulgation de la loi Bertrand du 29 décembre 2011 . L’Agence française de sécurité sanitaire du médicament et des produits de santé (AFSSAPS) n'existe plus; elle a été remplacée par l'ANSM. Le recours à l'expertise a été renforcé en interne. Elle s'organise en huit directions produits (correspondant à des sphères thérapeutiques). En outre, des directions métiers ont été créées ; parmi celles-ci figure la direction de l'évaluation que je dirige. La commission d'AMM ayant disparu, quatre commissions ont été créées. Elles ont pour mission d'éclairer la décision de l'Agence. L'une des commissions concerne l'évaluation initiale du rapport bénéfice/risque, dans le cadre de laquelle sont programmées notamment les RTU et les ATU cohorte. Les ordres du jour et les comptes rendus des commissions, ainsi que les vidéos sont disponibles sur le site Internet de l'Agence. Les associations de patients, dont notamment quatre représentants du monde des maladies rares, sont présentes au sein de ces commissions.

Les experts externes sont nommés par le directeur de l'Agence. Leur déclaration publique d'intérêt est disponible sur le site de l'Agence. L'organisation du recours à l'expertise externe se fait par ailleurs via des groupes de travail ou via de l'expertise externe ponctuelle.

\section{LIENS D'INTÉRÊT}

M.C. Borrelly déclare avoir des liens permanents avec l'entreprise Octapharma France SAS. Les autres auteurs déclarent n'avoir aucun lien d'intérêt concernant les données publiées dans cet article.

\section{ÉCHANGES AVEC LA SALLE}

\section{De la salle}

Les demandes de RTU par les centres de référence maladies rares sont nombreuses. Combien de demandes ont été acceptées? Les dossiers « refusés » feront-ils l'objet d'un retour et d'explications? 


\section{Cécile Delval}

Une majorité de dossiers sont refusés par manque d'évidence de données qui nous permettraient de démarrer l'instruction. Nous vous présenterons un bilan plus détaillé au fur et à mesure de l'analyse des signalements.

\section{De la salle}

Est-il plus facile d'obtenir une AMM pour les médicaments des maladies rares ? Êtes-vous plus souple sur les critères d'éligibilité ?

\section{Cécile Delval}

L'ANSM instruit un médicament dans les maladies rares comme tout autre médicament. Nous répondons bien sûr aux exigences de données et d'évidence, tout en ayant un certain nombre de prérequis liés au monde des études réalisées avec de petits effectifs.

\section{De la salle}

Pourquoi les RTU ne font-ils pas l'objet de remboursement puisque I’ANSM juge le bénéfice/risque pour le patient?

\section{Anne d'Andon}

Le remboursement dérogatoire des RTU est prévu. Après l'évaluation réalisée par l'ANSM pour recommander cet usage temporaire de médicaments utilisés en dehors des indications de l'AMM, nous nous prononcerons sur le remboursement. Jusqu'à présent, nous n'avons pas eu l'occasion de le faire puisque auparavant le dispositif était différent (la HAS, initiatrice, sollicitait I'AFSSAPS pour se prononcer d'une part sur la balance bénéfice/risque, et d'autre part, avec l'avis de I'AFSSAPS, sur un remboursement dérogatoire). Le nouveau dispositif est séquentiel. L'ANSM propose une RTU; la HAS propose ensuite un remboursement. La sortie des premières RTU devrait être imminente.

\section{De la salle}

Y a-t-il une réflexion sur la gestion des patients, qui seront sous un traitement en RTU, au terme de la durée maximale de la RTU si le laboratoire ne peut pas demander une AMM en l'absence de données cliniques suffisantes au regard du niveau d'exigence de I'AMM?

\section{Annie Lorence}

Les RTU seront octroyées pour trois ans. À l'issue de cette période, l'intérêt du médicament sera réévalué en tenant compte des alternatives et données nouvelles dans l'indication.

\section{Chrystel Jouan-Flahault}

L'octroi de RTU peut-il être renouvelé ?

\section{Annie Lorence}

Les textes réglementaires ne mentionnent pas le terme «renouvelable ». Au cours de la période des trois ans, de nouvelles alternatives pourraient apparaître et alors la RTU pourrait être abrogée avant l'échéance.

\section{Chrystel Jouan-Flahault}

S'il n'y a pas de nouvelles alternatives, un délai de trois ans est en réalité très court.

\section{Annie Lorence}

II me semble que le terme « renouvelable » a fait l'objet de discussions, mais il n'a finalement pas été retenu dans le décret.

\section{Christophe Duguet}

Le texte ne dit pas que la RTU est donnée pour trois ans, sans préciser qu'elle est renouvelable. Le texte ne dit pas non plus qu'un dossier complet d'une nouvelle RTU ne peut pas être refait.

\section{De la salle}

Un programme national de cohorte visant à mieux comprendre l'histoire de la maladie devrait-il aussi se positionner sur des cohortes pour avoir des données consolidées sur les utilisations hors AMM de médicaments?

L'utilisation de médicaments «off licence » pour des enfants atteints de maladies rares génère des problématiques de formulation et de présentation. Des «incentives » sont-elles envisagées en France pour permettre des essais cliniques sur des protocoles adaptés aux enfants?

\section{Anne d'Andon}

Des registres de bonne qualité permettent de collecter des informations servant à mesurer des interventions thérapeutiques, en vue d'une nouvelle RTU ou d'une AMM. Par ailleurs, je précise que la HAS ne finance pas ces registres.

\section{Christophe Duguet}

Comme nous sommes dans une situation de non-droit autour du hors AMM, aucun dispositif n'a été prévu pour financer des outils permettant de suivre quelque chose d'«illégal ». C'est pourquoi il est nécessaire d'inventer un nouveau système qui permettra, de manière légale et moyennant un certain nombre de contraintes, de suivre et de mettre dans des cohortes des patients qui sont hors AMM et hors RTU. Cette évolution réglementaire est indispensable pour que des financements publics soient alloués au suivi et à la réalisation d'essais cliniques. Ceuxci sont notamment nécessaires pour un grand nombre de molécules anciennes et «génériquées 》. Les industriels ne vont pas financer ces essais cliniques puisqu'ils n'auront aucun retour garanti sur leur investissement. D'aucuns estiment que ces essais cliniques doivent être financés par le programme hospitalier de recherche clinique (PHRC). 
Or, celui-ci n'a pas les moyens de financer quelques centaines d'essais cliniques, même programmés sur plusieurs années.

Dans ce contexte, il est indispensable de mettre en place un dispositif permettant spécifiquement de financer des essais, des mises en cohorte, etc. II peut également être envisagé d'inventer un système donnant-donnant. Les essais cliniques pourraient alors faire l'objet d'appels d'offres. L'industriel retenu aurait alors la garantie que le prix du médicament lui permettra de se rembourser et de faire une marge sur le risque pris. Il y a urgence à trouver des solutions, car il en va de la crédibilité du système.

\section{Brigitte Chabrol}

Le problème du hors AMM en pédiatrie est au cœur de notre préoccupation, pour les maladies rares comme pour les autres maladies. Nous venons de réaliser une enquête au sein du CHU Timone. Durant une semaine, nous avons analysé 835 ordonnances de sortie, venant de services très spécialisés et des urgences pédiatriques. $45 \%$ d'entre elles étaient hors AMM, principalement du fait de l'âge du patient. Pour certaines molécules, il existe un argumentaire pour l'adulte ou le grand enfant. Comment une AMM pourra-t-elle être accordée pour le petit enfant? Ni la RTU, ni les projets d'investigation pédiatrique ne pourront élargir cela. En outre, il faut savoir que les caisses ne remboursent plus les patients qui se voient prescrire des médicaments hors AMM. Dans le domaine des maladies rares, il est nécessaire d'aller justifier chaque semaine toutes nos ordonnances, qui sont ensuite payées par un fonds spécifique. Cela fait un an et demi que cette situation perdure. II est indispensable de s'attaquer à ce domaine pédiatrique pur, car nous ne pouvons pas continuer ainsi. II nous est demandé de faire des argumentaires et des essais cliniques, alors que nous ne pouvons pas les réaliser.

\section{Marie-Christine Borrelly}

Certains centres de référence sont en train de mettre en place des registres. Ainsi le registre français des tolérances immunes vient d'être finalisé. II a été adressé aux laboratoires avec des conventions. Nous nous engageons à soutenir et à travailler main dans la main avec les autorités et les centres de référence.

\section{De la salle}

Au-delà de l'âge, la durée est également importante dans le domaine de la pédiatrie. La combinaison de ces deux paramètres rend très complexe le montage des dossiers. Par ailleurs, je pense que les centres de référence ont été sollicités pour de multiples raisons. En outre, ils n'ont sans doute pas été en mesure de répondre à l'ensemble de vos questions. Nous allons leur dire qu'ils doivent essayer de vous répondre.

\section{Cécile Delval}

C'est pour cela que nous avons décidé de les relancer. 